\title{
PTEN, PIK3CA, p-AKT, and p-p70S6K Status
}

\section{Association with Trastuzumab Response and Survival in Patients with HER2-Positive Metastatic Breast Cancer}

\author{
Francisco J. Esteva, ${ }^{\text {† }}$ Hua Guo, ${ }^{\star}$ Siyuan Zhang, ${ }^{\text {* }}$ \\ Cesar Santa-Maria, ${ }^{\ddagger}$ Steven Stone, ${ }^{\ddagger}$ \\ Jerry S. Lanchbury, ${ }^{\ddagger}$ Aysegul A. Sahin, ${ }^{\S}$ \\ Gabriel N. Hortobagyi, ${ }^{\dagger}$ and Dihua $\mathrm{Yu}^{* \pi}$ \\ From the Departments of Molecular and Cellular Oncology,* \\ Breast Medical Oncology, ${ }^{\dagger}$ and Pathology, ${ }^{\S}$ the University of \\ Texas M.D. Anderson Cancer Center, and the Cancer Biology \\ Program, "the University of Texas Graduate School of Biomedical \\ Sciences at Houston, Houston, Texas; and Myriad Genetics, ${ }^{\ddagger}$ Salt \\ Lake City, Utah
}

Phosphatase and tensin homolog (PTEN) is a key modulator of trastuzumab sensitivity in HER2-overexpressing breast cancer. Because PTEN opposes the downstream signaling of phosphoinositide 3-kinase (PI3K), we investigated the role of PTEN and other components of the PI3K pathway in trastuzumab resistance. We analyzed the status of PTEN, p-AKTSer473, and p-p70S6K-Thr389 using immunohistochemistry. PIK3CA mutation status was analyzed by direct sequencing. Primary tumor tissue was available from 137 patients with HER2-overexpressing metastatic breast cancer who had received trastuzumabbased chemotherapy. We observed that each of the four biomarkers alone did not significantly correlate with trastuzumab response, whereas PTEN loss alone significantly correlated with shorter survival times $(P=0.023)$. PI3K pathway activation, defined as PTEN loss and/or PIK3CA mutation, was associated with a poor response to trastuzumab $(P=0.047)$ and a shorter survival time $(P=0.015)$. PTEN loss was significantly associated with a poor response to trastuzumab $(P=0.028)$ and shorter survival time $(P=$ $0.008)$ in patients who had received first-line trastuzumab and in patients with estrogen receptor- $(P=$ $0.029)$ and progesterone receptor-negative tumors $(P=0.033)$. p-AKT-Ser 473 and p-p70S6K-Thr389 each had a limited correlation with trastuzumab response. When these markers were combined with PTEN loss, an increased correlation with patient outcome was observed. In conclusion, PI3K path- way activation plays a pivotal role in trastuzumab resistance. Our findings may facilitate the evaluation of tumor response to trastuzumab-based and targeted therapies. (Am J Pathol 2010, 177:1647-1656; DOI: 10.2353/ajpath.2010.090885)

Human epidermal growth factor receptor 2 (HER2) is overexpressed in $20 \%$ to $25 \%$ of invasive breast cancers. Patients with HER2-overexpressing tumors experience a shorter time to relapse and shorter overall survival than patients with tumors of normal HER2 levels., ${ }^{1,2}$ HER2 overexpression can lead to activation of many downstream signaling molecules, including Ras-Gap, Src, phosphoinositide 3-kinase (PI3K)/AKT, and many other signaling events. ${ }^{3,4}$ Trastuzumab (Herceptin; Genentech, $\mathrm{CA}$ ), a humanized monoclonal antibody that directly targets the extracellular domain of HER2, has a remarkable therapeutic efficacy in treating patients with HER2-expressing metastatic breast cancer $(\mathrm{MBC})^{5}$ and patients with HER2-positive early-stage disease in adjuvant settings. 6,7 Trastuzumab treatment, when combined with chemotherapy, resulted in a significant improvement in patients' response rate, time to progression, and duration

Supported in part by NIH grants P30-CA 16672 (MDACC), M.D. Anderson Cancer Center Breast SPORE P50 CA116199 (project 4), and the Breast Cancer Research Foundation (F.J.E); RO1-CA112567, DOD Center of Excellence grant subproject W81XWH-06-2-0033, DOD Synergistic Award W81XWH-08-1-0712, and Susan G. Komen Breast Cancer Foundation Promise grant KG091020 (D.Y.). D.Y. is the Hubert L. \& Olive Stringer Distinguished Chair in Basic Science at M.D. Anderson Cancer Center.

F.J.E. and H.G. contributed equally to this study.

Accepted for publication June 16, 2010.

Supplemental material for this article can be found on http://ajp. amjpathol.org.

Current address of H.G.: Department of Pathology, Peking University First Hospital, 1\# Da Hong Luo Chang Street, Xicheng District, Beijing 100034, China.

Address reprint requests to Dihua Yu, M.D., Ph.D., The University of Texas M. D. Anderson Cancer Center, 1515 Holcombe Boulevard, Houston, TX 77030. E-mail: dyu@mdanderson.org. 
of response. ${ }^{8}$ The underlying mechanisms of trastuzumab's antitumor activities include, but are not limited to, inducing antibody-dependent cellular cytotoxicity, ${ }^{9}$ inhibiting HER2 extracellular domain cleavage, ${ }^{10}$ activating phosphatase and tensin homolog (PTEN) ${ }^{11}$ and inhibiting PI3K/AKT survival signaling. ${ }^{12}$ However, the overall response rate to trastuzumab is low, and almost half of patients with HER2-positive breast cancer exhibit an initial resistance to trastuzumab-based therapy. ${ }^{11,13}$ Despite the large amounts of preclinical and clinical data, the causes of trastuzumab resistance are still poorly understood. ${ }^{14}$

The PI3K pathway, downstream of HER2, plays a central role in regulating a number of cellular processes, such as apoptosis, migration, angiogenesis, cell proliferation, and glucose metabolism, and it is involved in trastuzumab resistance. ${ }^{15,16}$ PI3K phosphorylates phosphatidylinositols on the cell membrane, generating phosphatidylinositol-3,4,5-trisphosphate (PIP3) from phosphatidylinositol-4,5-bisphosphate (PIP2). Then, at the cell membrane, PIP3 recruits protein kinases and activates protein kinase $B(P K B) / A K T .{ }^{17}$ In breast cancer cells, HER2 overexpression can lead to activation of the PI3K/AKT pathway. ${ }^{18}$ The activation of AKT and its downstream signaling have been demonstrated to inhibit cell cycle arrest and block trastuzumab-mediated apoptosis. ${ }^{12}$ AKT phosphorylation and AKT kinase activities were found to be increased in trastuzumab-resistant cells, derived from BT474 HER2-overexpressing breast cancer cells, when compared with parental cells. ${ }^{19}$ These data provide insight into the trastuzumab-resistance mechanism of PI3K/AKT signaling. ${ }^{15}$

Aberrations in the components of the PI3K pathway have been reported in most solid tumors, including breast cancer. ${ }^{16}$ PTEN is a tumor suppressor that dephosphorylates the D3 position of PIP3 and inhibits the PI3K/AKT pathway. ${ }^{20}$ Loss of PTEN function as a result of mutation, deletion, or promoter methylation has been reported in nearly $50 \%$ of breast cancers. ${ }^{11}$ In addition, the gene encoding one of the PI3K catalytic subunits, $\mathrm{p} 110 \alpha$ (PIK3CA), has been found to be mutated in about $25 \%$ of breast cancers. ${ }^{21,22,23}$ Most of the reported mutations are localized to hotspots in exons 9 and 20 of the PIK3CA gene, which result in increased PI3K pathway signaling. ${ }^{22,24}$ We previously discovered that PTEN activation is a novel mechanism of trastuzumab antitumor function, and PTEN loss confers trastuzumab resistance in HER2overexpressing breast cancer cells. ${ }^{11}$ PTEN loss significantly predicted poor response to trastuzumab-based therapy in a small cohort of HER2-positive patients with MBC. ${ }^{11}$ Later, it was reported that both low PTEN levels and PI3K-activating PIK3CA mutations contribute to trastuzumab resistance in HER2-overexpressing breast cancer. ${ }^{25,26}$ PTEN loss or PIK3CA mutations, which indicate activation of the PI3K pathway, are considered as markers for poor response to trastuzumab in patients with HER2-overexpressing breast cancer. ${ }^{25}$ On the other hand, some studies found no correlation between PTEN expression and trastuzumab response or survival in patients with HER2-positive breast cancer. ${ }^{27,28}$ These contradictory findings prompted us to further investigate the association between PTEN status and clinical outcomes in a large cohort of patients with MBC who were treated with trastuzumab-based therapy. We tested the hypothesis that a comprehensive assessment of PI3K pathway activation status provides biomarkers that can identify patients who may not benefit from trastuzumab-based therapy.

\section{Materials and Methods}

\section{Patients and Tissue Samples}

HER2-positive tumor samples were obtained from 137 eligible patients at the University of Texas M. D. Anderson Cancer Center under an institutional review board-approved protocol. All of the patients, who subsequently developed MBC, were treated with trastuzumab-based therapy between 1998 and 2006. Tumors that scored 3+ on immunohistochemical (IHC) analysis of HER2 and/or had an amplified HER2 gene detected by fluorescence in situ hybridization (FISH) were defined as HER2 positive. PTEN, p-AKT, and p-p70S6K status, determined by IHC analysis, were evaluated on serial slides and scored on all tissue samples, except for one sample on which pAKT scoring could not be obtained.

\section{IHC Analysis}

IHC staining was performed on $4-\mu \mathrm{m}$ slices of formalinfixed paraffin-embedded (FFPE) tissue sections with primary antibodies against PTEN (138G6, 1:50; Cell Signaling Technology, Danvers, MA), p-AKT (Ser473, 736E11, IHC specific, Cell Signaling; 1:50), and p-p70S6K (Thr389, 1A5, Cell Signaling, 1:700). To validate the specificity of PTEN antibodies, Western blotting was performed first using both BT474 control cells and BT474 PTEN-deficient cell lines (by shRNA knockdown). Then mouse xenograft tumors derived from above cell lines were embedded in paraffin and used as negative and positive control for $\mathrm{IHC}$ staining. After comparing four PTEN antibodies from different companies (Cell Signaling; R\&D Systems, Minneapolis, MN; Cascade Bioscience, Winchester, MA; and Dako, Carpinteria, CA), we found that the antibody from Cell Signaling is the most specific in our studies (data not shown). The p-AKT and p-p70S6K stainings were validated by Western blotting and other studies, as previously described. ${ }^{29,30}$ After deparaffinization and rehydration, slides were subjected to heat-induced epitope retrieval in $10 \mathrm{mmol} / \mathrm{L}$ citrate buffer ( $\mathrm{pH}$ 6.0) for $\mathrm{p}$-p70S6K staining or $1 \mathrm{mmol} / \mathrm{L}$ EDTA $(\mathrm{pH}$ 8.0) for PTEN and $\mathrm{p}-\mathrm{AKT}$ staining in a hot water bath $\left(95^{\circ} \mathrm{C}\right)$ for 20 minutes. Endogenous peroxidase activity was blocked for 15 minutes in $0.3 \%$ hydrogen peroxide. After blocking with $1 \%$ goat serum for 1 hour at room temperature, the sections were incubated with primary antibodies for at least 18 hours at $4^{\circ} \mathrm{C}$ overnight. Immunodetection was performed using the LSAB2 kit (Dako), 3-3'-diaminobenzidine was used for color development, and hematoxylin was used for counterstaining. Positive control slides (from BT474 xenograft tumors) and negative control slides (without primary antibody) were included within each batch for staining. 


\section{Evaluation of IHC Staining}

The PTEN, p-AKT, and p-p70S6K expression levels were evaluated based on the staining intensity (SI) and percentage of positive cells (PP) within the whole tissue section. PTEN was scored semiquantitatively using the immunoreactive score (IRS), which was calculated as follows: IRS $=\mathrm{SI} \times \mathrm{PP}$. SI was defined as $0=$ negative, $1=$ weak, $2=$ moderate, and $3=$ strong. $\mathrm{PP}$ was scored as $0=1 \% ; 1=1 \%-10 \% ; 2=11 \%-50 \%$; $3=51 \%-$ $80 \%$; and $4=>80 \%$ positive cells. PTEN loss was defined as an IRS of 3 or less. ${ }^{11}$ The p-AKT and p-p70S6K stainings were evaluated by an $\mathrm{H}$ score, ${ }^{31}$ which was calculated by multiplying the PP by the corresponding SI ( 1 = weak, 2 = moderate, and 3 = strong $)$, giving a maximum score of $300(100 \% \times 3)$. H scores $>50$ were considered positive.

\section{PIK3CA PCR, Sequencing, and Mutational Analysis}

We isolated genomic DNA from FFPE tumor samples for PIK3CA mutation screening from 90 patients. The other 47 tumors are very small in size and did not allow analysis of PIK3CA mutations. PIK3CA mutation screening was performed by using the QIAamp DNA FFPE tissue kit (Qiagen, Valencia, CA) according to the kit protocol. First, the FFPE slides were stained with hematoxylin and eosin and checked by a pathologist to identify the tumor region. After deparaffinization, most of the tumor was cut out from the slides by a razor blade. For a few samples, dissection was aided by laser capture microscopy owing to the dispersion of the tumor cells. For laser capture, we used a PALM MicroBeam Microscope for Laser Micromanipulation (Carl Zeiss, Inc., Thornwood, NY) with HVD30 Hitachi camera capture system (Tokyo, Japan). The microscope contains built-in class IIIB and class IV lasers. The equipment was run using PALM Robo Software 4.0.0.10 (Carl Zeiss), and all laser capture was directed by a pathologist.

The isolated genomic DNA was diluted to $3 \mathrm{ng} / \mu \mathrm{l}$ with TE buffer (10 mmol/L Tris- $\mathrm{HCl}, 0.1 \mathrm{mmol} / \mathrm{L}$ EDTA [pH 8.0]) and sent to a DNA sequencing facility for sequencing of exons 9 and 20 of PIK3CA. For each sample, we submitted $6 \mathrm{ng}$ of genomic DNA. The DNA was amplified using the primer pair 5'-GTTTTCCCAGTCACGACGTATTGAAAATGTATTTGCTTTTTC and 5'-GGAAACAGCTATGACCATAACATGCTGAGATCAGCCAAAT for exon 9 and the primer pair 5'-GTTTTCCCAGTCACGACGATTAACATCATTTGCTCCAAAC and 5'-AGGAAACAGCTATGACCATGCTGTTTAATTGTGTGGAAGAT for exon 20. The primers also contained M13 forward and reverse tails to facilitate sequencing. After amplification, the DNA sequence was determined on a Mega BASE 4500 (GE Health care, Waukesha, WI) using dye-primer chemistry, as previously described. ${ }^{32}$ The observed mutations were confirmed by two independent amplification and sequencing reactions. The success rate was 60\% (55/90).

\section{Evaluation of Trastuzumab Response and Statistical Analyses}

Response to trastuzumab-based therapy was evaluated every 8 to 12 weeks using the response evaluation criteria in solid tumors (RECIST) with modifications. ${ }^{33} \mathrm{~A}$ complete response (CR) was defined as the complete disappearance of all radiographically or visually apparent tumors for at least 4 weeks. A partial response (PR) was defined as $a \geq 30 \%$ decrease in the maximum diameter of measurable metastatic lesions for at least 4 weeks, with no new lesions appearing. Stable disease (SD) was defined as no change in the size of measurable lesions $\geq 20 \%$ and no appearance of new lesions. Progressive disease (PD) was defined as the appearance of any new lesion or an increase in the size of the existing lesions $\geq 20 \%$. In this study, response was defined as a CR, PR, or SD longer than 6 months (clinical benefit $=\mathrm{CR}+\mathrm{PR}+$ $\mathrm{SD}$ ). Overall survival (OS) was defined as the time from initiation of trastuzumab treatment to the patient's death or the final day of follow-up.

The relationship between the expression of the biomarkers and response to trastuzumab and clinicopathologic features was analyzed using Fisher's exact test and the $\chi^{2}$ test. Survival analyses were performed using KaplanMeier curves with log-rank tests. A $P$ value $<0.05$ (twosided) was considered to be statistically significant. All statistical analyses were performed using the SPSS 16.0 program (SPSS, Chicago, IL).

\section{Results}

\section{Demographic and Pathological Characteristics of the Study Population}

The study population comprised 137 patients with HER2positive MBC who received trastuzumab treatment. The patients' clinical characteristics are summarized in Table 1. The median age at first diagnosis was 44 years (range, 20-73 years). Of the 137 patients, 123 (89.8\%) were initially diagnosed with invasive ductal carcinoma. Most patients were white (104, 75.9\%); 54 patients (39.4\%) had estrogen receptor (ER)-positive tumors, and 44 $(32.1 \%)$ had progesterone receptor (PR)-positive tumors. The median disease-free survival time before developing metastatic disease was 18 months (range, 0-169 months), and most patients (51.8\%) had multiple distant metastases. Among the 137 patients, the three most frequent first metastatic sites were the liver $(65,47.4 \%)$, bone $(60,43.8 \%)$, and lungs $(52,38.0 \%)$. During followup, 45 patients (32.8\%) developed brain metastases, of which $31(31 / 45=68.9 \%)$ were diagnosed with brain metastasis after initiation of Trastuzumab-based therapy.

The patients' demographic characteristics had no relationship with response to trastuzumab or overall survival after trastuzumab treatment, except for race. Black patients had the shortest OS after starting trastuzumab $(P=0.014)$ compared with other groups. Information on the patients' trastuzumab-based therapy and patients' clinical outcomes were summarized in Table 2. Of the 
Table 1. Clinicopathological Characteristics of 137 Metastatic Breast Cancer Patients Treated with TrastuzumabBased Therapy

\begin{tabular}{|c|c|}
\hline Characteristic & $\mathrm{n}(\%)$ \\
\hline \multicolumn{2}{|l|}{$\begin{array}{l}\text { Age at diagnosis (median } 44 \text { years, range } \\
20-73 \text { ) }\end{array}$} \\
\hline$<50$ years & $89(65)$ \\
\hline$\geq 50$ years & $48(35)$ \\
\hline \multicolumn{2}{|l|}{ Nuclear grade } \\
\hline I & 0 \\
\hline II & $22(16)$ \\
\hline III & $106(77.4)$ \\
\hline Unknown & $9(6.6)$ \\
\hline \multicolumn{2}{|l|}{ Histologic type } \\
\hline Invasive ductal carcinoma & $123(89.8)$ \\
\hline Invasive mixed ducal/lobular carcinoma & $8(5.8)$ \\
\hline Invasive lobular carcinoma & $2(1.5)$ \\
\hline Inflammatory carcinoma & $1(0.7)$ \\
\hline Invasive micropapillary carcinoma & $1(0.7)$ \\
\hline Breast cancer, NOS & $2(1.5)$ \\
\hline \multicolumn{2}{|l|}{ Race/ethnicity } \\
\hline White & $104(75.9)$ \\
\hline Black & $8(5.8)$ \\
\hline Hispanic & $17(12.4)$ \\
\hline Asian & $6(4.4)$ \\
\hline Others & $2(1.5)$ \\
\hline \multicolumn{2}{|l|}{ ER status } \\
\hline Positive & $56(40.9)$ \\
\hline Negative & $78(56.9)$ \\
\hline Unknown & $3(2.2)$ \\
\hline \multicolumn{2}{|l|}{ PR status } \\
\hline Positive & $45(32.8)$ \\
\hline Negative & $85(62.0)$ \\
\hline Unknown & $7(5.1)$ \\
\hline \multicolumn{2}{|l|}{ First site of metastasis } \\
\hline Liver & $65(47.4)$ \\
\hline Bone & $60(43.8)$ \\
\hline Lung & $52(38)$ \\
\hline Brain & $9(6.6)$ \\
\hline \multicolumn{2}{|l|}{ Brain metastasis } \\
\hline $\begin{array}{l}\text { Total cases from time of original } \\
\text { diagnosis }\end{array}$ & $45(32.8)$ \\
\hline
\end{tabular}

ER indicates estrogen receptor; PR, progesterone receptor.

137 patients, 87 (63.5\%) used trastuzumab treatment as first-line therapy, and 91 (66.4\%) had received no prior chemotherapy. Eighty-two patients (59.9\%) responded to trastuzumab-based therapy (CR + PR + SD), with a median OS of 30 months (range, 2-84 months). However, 55 patients $(40.1 \%)$ were resistant to trastuzumab (PD), with a median OS of only 13 months (range, 1-49, months; $P<0.001$ compared with $\mathrm{CR}+\mathrm{PR}+\mathrm{SD}$ group).

\section{PTEN and PIK3CA Status and Their Relationship with the Patients' Clinicopathologic Characteristics}

To determine PTEN protein expression in breast tumors, we first validated the specificity and sensitivity of PTEN antibodies for IHC (see Materials and Methods and Supplemental Figure S1, A and B at http://ajp.amjpathol.org). As shown in Figure 1A, PTEN expression in tumors was heterogeneous and PTEN had both cytoplasmic and nuclear localizations. A strong PTEN staining in fibroblasts cells was used as internal positive controls along with paraffin sections containing both control.shRNA and
Table 2. Trastuzumab-Based Therapy Information and Clinical Outcomes of the 137 Metastatic Breast Cancer Patients

\begin{tabular}{cc}
\hline \multicolumn{1}{c}{ Variable } & Description \\
\hline Ttzm-based therapy & \\
Ttzm single agent & $16(11.7 \%)$ \\
Ttzm + navelbine & $30(21.9 \%)$ \\
Ttzm + taxane-based & $76(55.5 \%)$ \\
chemotherapy & \\
Ttzm + other therapy & $15(10.9 \%)$ \\
Lines of Ttzm-based therapy & $87(63.5 \%)$ \\
First-line & $26(19.0 \%)$ \\
Second-line & $24(17.5 \%)$ \\
Other & $91(66.4 \%)$ \\
Prior chemotherapy & $39(28.5 \%)$ \\
Without & $7(5.1 \%)$ \\
With & \\
Unknown & $82(59.9 \%)$ \\
Response to Ttzm-based & $55(40.1 \%)$ \\
therapy & \\
CR + PR + SD & $97(70.8 \%)$ \\
PD & $40(29.2 \%)$ \\
Outcome & $23 m($ range $1-84 \mathrm{~m})$ \\
Dead & \\
Alive & $30 \mathrm{~m}($ range $2-84 \mathrm{~m})$ \\
Median time of overall & \\
survival from Ttzm-based & \\
therapy start date & \\
Patients responsive (CR + & \\
PR + SD) to therapy & \\
Patients resistant (PD) to & (range 1-49 m) \\
therapy &
\end{tabular}

Ttmz indicates trastuzumab; CR, complete response; PR, partia response; SD, stable disease; $\mathrm{PD}$, progressive disease.

PTEN.shRNA xenograft tumors as positive and negative controls (Figure 1B). Based on our previous studies, we used an IRS score $\leq 3$ as the criterion for PTEN loss or reduced PTEN expression. ${ }^{11}$ Using this cut-off, we observed loss of PTEN expression in 71 tumors $(51.8 \%)$ in the 137 patients' HER2-positive MBC (Table 3). The patients' clinico-pathological characteristics were not significantly associated with PTEN status (data not shown).

We performed PIK3CA mutation analysis on 90 of the 137 samples. The other 47 tumors are very small in size and did not allow analysis of PIK3CA mutations. The success rate (as measured by getting reliable sequence at $\mathrm{E} 542$, E545, and $\mathrm{H} 1047)$ was $60 \%$ (55/90). We also confirmed the findings by PCR analyses. We found that 12 tumors (21.8\%) had PIK3CA mutations among the 55 patients with HER2-overexpressing breast cancer (Table $3)$. The most common mutation was an H1047R mutation in exon 20 (7/12, 12.7\%). Four of the 12 tumors had an E545K mutation, and one had an E542K mutation. PIK3CA mutation status had no association with any of the clinic-pathological parameters $(P>0.05$, data not shown).

\section{Relationship Between PTEN and PIK3CA Mutation Status and Patients' Clinical Outcomes after Trastuzumab-Based Therapy}

We found that among the 137 patients, those who had tumors with PTEN loss (IRS $\leq 3$ ) were more likely to be resistant to trastuzumab-based therapy than those who 
A

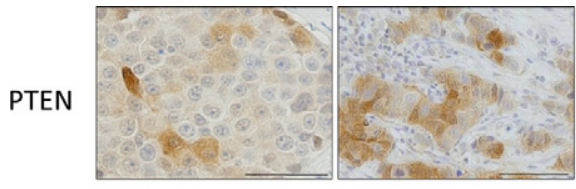

B

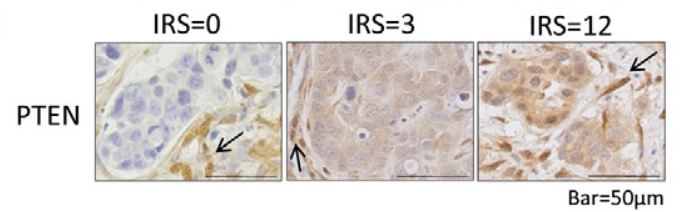

C
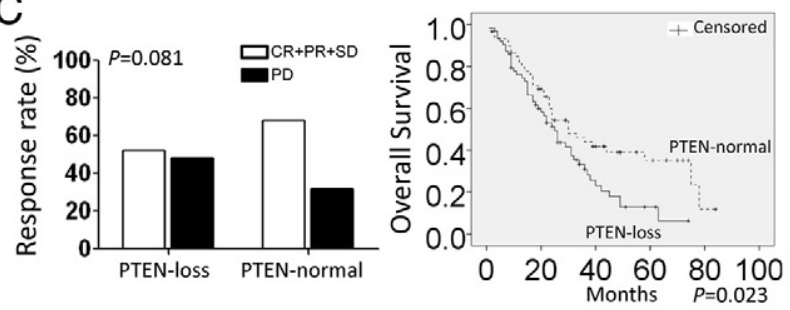

D Activation of PI3K pathway $\quad$ CR+PR+SD PD Total PTEN-loss or/and PIK3CA mutation

\begin{tabular}{lccc} 
Yes & 18 & 18 & 36 \\
No & 15 & 4 & 19 \\
Total & 33 & 23 & 55 \\
\hline
\end{tabular}

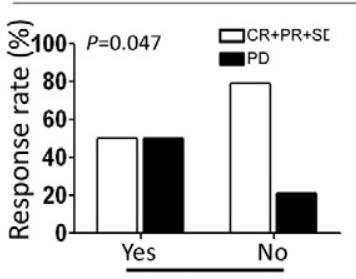

PTEN-loss or/and PIK3CA mutation

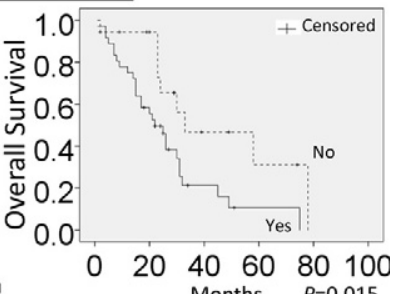

Months $P=0.015$

Figure 1. Activation of the PI3K pathway was more significantly associated with trastuzumab resistance than PTEN status alone. A: Heterogeneous expression of the PTEN protein in invasive ductal carcinoma lesions examined by IHC in HER2-positive MBC tumors. B: Representative PTEN IHC staining (IRS $=0,3,12$ ). Arrows point to fibroblasts and endothelial cells that showed high expression of PTEN, which served as an internal positive control. C: Poor response $(P=0.081)$ and significantly shorter OS $(P=$ 0.023 ) after trastuzumab-based therapy in patients with PTEN loss alone (PTEN loss defined as IRS $\leq 3)$. D: Significantly poorer response $(P=0.047)$ and shorter OS $(P=0.015)$ after trastuzumab-based therapy in patients with PI3K pathway activation (defined as PTEN loss and/or PIK3CA mutation). Scale bar $=50 \mu \mathrm{m}$

had PTEN-normal tumors, but PTEN loss itself was not significantly correlated with response $(P=0.081$; Table 3; Figure 1C). The Kaplan-Meier curve demonstrated a significantly shorter survival for patients with PTEN-deficient tumors $(P=0.023$; Figure $1 \mathrm{C})$. PIK3CA mutation was not significantly associated with either a response to trastuzumab-based therapy $(P=1.000$; Table 3$)$ or an improved OS time ( $P=0.172)$; patients with the PIK3CA mutation seemed to have a slight trend toward shorter OS times (Table 3; also see Supplemental Figure S2 at http://ajp.amjpathol.org).

Because both PTEN loss and PIK3CA mutations lead to PI3K pathway activation and because activated PI3K signaling were reported to contribute to trastuzumab resistance, we analyzed trastuzumab response based on a combination of PTEN and PIK3CA mutation status. Patients having tumors with PI3K pathway activation (defined as PTEN loss and/or PIK3CA mutation) were more
Table 3. Associations between PTEN and PIK3CA Status and Response to Trastuzumab-Based Therapy

\begin{tabular}{lcccc}
\hline \multicolumn{1}{c}{ Variables } & $\begin{array}{c}\text { CR }+ \text { PR } \\
+ \text { SD }\end{array}$ & PD & Total (\%) & $\begin{array}{c}P \\
\text { Value }\end{array}$ \\
\hline PTEN & & & & \\
$\quad$ PTEN loss & 37 & 34 & $71(51.8)$ & 0.081 \\
$\quad$ PTEN normal & 45 & 21 & $66(48.2)$ & \\
$\quad$ Total & & & $137(100)$ & \\
PIK3CA mutation & & & & \\
$\quad+$ & 7 & 5 & $12(21.8)$ & 1.000 \\
$\quad$ & 26 & 17 & $43(78.2)$ & \\
$\quad$ Total & & & $55(100)$ & \\
\hline
\end{tabular}

CR indicates complete response; PR, partial response; SD, stable disease; PD, progressive disease.

resistant to trastuzumab-based therapy (PD) and received less clinical benefit $(C R+P R+S D)$ than patients having tumors without PI3K pathway activation ( $P=$ 0.047; Figure 1D). In addition, patients having tumors with an activated PI3K pathway had a significantly shorter OS $(P=0.015)$ than patients whose tumors do not have PI3K pathway activation (Figure 1D).

\section{Correlation between $p-A K T$ and p-p70S6K Status and Their Relationship with Patients' Clinical Outcomes after Trastuzumab-Based Therapy}

Because PTEN loss and PIK3CA mutations are involved in trastuzumab resistance and both can lead to activation of downstream signaling pathways, including increased $\mathrm{p}-\mathrm{AKT}$ and $\mathrm{p}-\mathrm{p} 70 \mathrm{S6K}$, we hypothesized that activation of signals downstream of the PI3KCA mutation and PTEN loss, such as increased p-AKT and p-p70S6K, would also correlate with trastuzumab resistance and serve as molecular markers for trastuzumab response. IHC staining showed that p-AKT had both cytoplasmic and nuclear localization, whereas p-p70S6K mainly had nuclear staining (Figure 2A). In some cases, tumors also displayed variable heterogeneous $\mathrm{p}-\mathrm{AKT}$ and $\mathrm{p}$-p70S6K staining similar to those of PTEN. We used the $\mathrm{H}$ score to quantify the staining, and positivity of $\mathrm{p}-\mathrm{AKT}(\mathrm{p}-\mathrm{AKT}+)$ and p-p70S6K (p-p70S6K+) were defined as $H \geq 50$. We detected p-AKT+ and p-p70S6K+ in 72 (52.9\%) and 93 $(67.9 \%)$ tumors, respectively. Notably, p-AKT and $\mathrm{p}-\mathrm{p} 70$ S6K status were significantly correlated $(P=0.001$; see Supplemental Table S1 at http://ajp.amjpathol.org). However, p-AKT and p-p70S6K status did not correlate with any of the patients' clinicopathologic characteristics described in Table 1 (data not shown).

The status of p-AKT and p-p70S6K alone showed no correlation with either trastuzumab response $(P=0.298$, $P=0.136$; see Supplemental Table S2 at http://ajp. amjpathol.org) or OS $(P=0.526, P=0.319$; see Supplemental Figure S3A and S3B at http://ajp.amjpathol.org). Combining p-AKT status and p-p70S6K status also had no correlation with either trastuzumab response or OS (data not shown). 
A

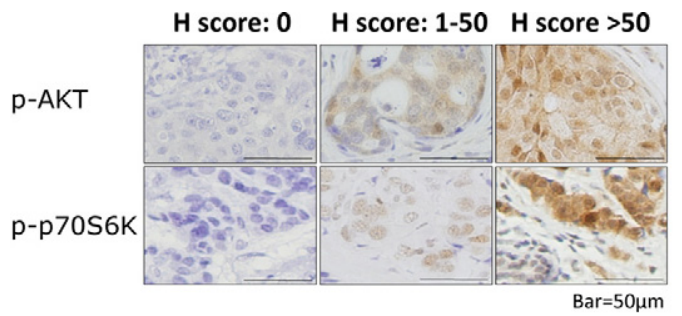

B
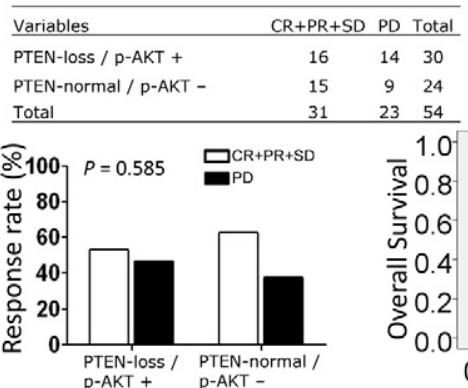

$23 \quad 54$

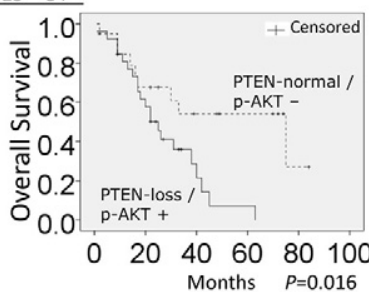

C
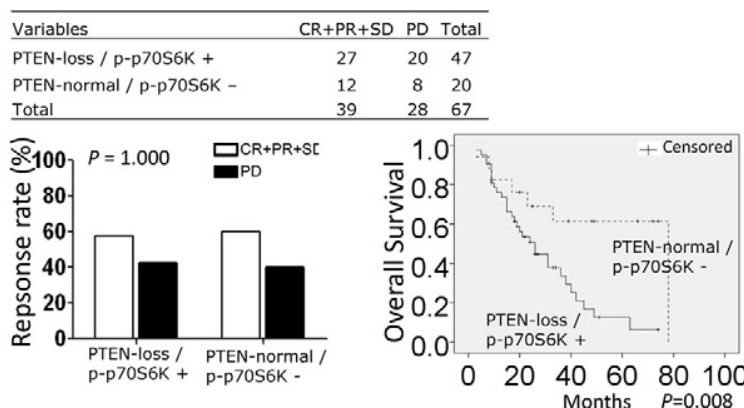

Figure 2. Combing either p-AKT + or p-p70S6K + with PTEN loss correlated with trastuzumab resistance. A: Representative IHC staining patterns of $\mathrm{p}$ AKT and p-p70S6K in HER2-positive MBC tumors. B: Combining p-AKT+ and PTEN loss was a more powerful marker of trastuzumab resistance than PTEN loss alone. Patients with PTEN-loss/p-AKT+ tumors showed worse responses and significantly shorter survival times $(P=0.016)$ after trastuzumab-based therapy. C: Combining p-p70S6K+ and PTEN loss was a more powerful marker of trastuzumab resistance than PTEN loss alone. Patients with PTEN-loss/p-p70S6K + tumors showed worse responses and significantly shorter OS $(P=0.008)$ after trastuzumab-based therapy. Scale bar $=50 \mu \mathrm{m}$

\section{p-AKT or p-p70S6K Combined with PTEN Status Correlated Well with Patients' Clinical Outcomes after Trastuzumab-Based Therapy}

We next examined whether combining either p-AKT+ or p-p70S6K + with PTEN loss correlated with trastuzumab resistance. The combination of PTEN loss with $\mathrm{p}-\mathrm{AKT}+$ was not significantly correlated with trastuzumab response ( $P=0.585$; Figure $2 \mathrm{~B})$. However, we observed a trend that patients with PTEN-loss/p-AKT+ tumors were more resistant to trastuzumab-based therapy than those with PTEN-normal/p-AKT- tumors. Moreover, the OS time after trastuzumab-based therapy was significantly shorter among those patients who had PTEN-loss/pAKT+ tumors than those with PTEN-normal/p-AKT- tumors $(P=0.016$; Figure $2 \mathrm{~B})$. Combined $\mathrm{p}-\mathrm{AKT}+$ and PTEN loss might be a more powerful marker of trastuzumab resistance than PTEN loss alone $(P=0.023$; Figure 1B). Similarly, combined PTEN and p-p70S6K status showed no correlation with trastuzumab response
$(P=1.000$; Figure $2 \mathrm{C})$, whereas patients who had PTENloss/p-p70S6K + tumors had a significantly shorter OS after trastuzumab-based therapy than those with PTENnormal/p-p70S6K- tumors $(P=0.008$; Figure $2 \mathrm{C})$. Thus, combined p-p70S6K + and PTEN loss might also be a more powerful marker of trastuzumab resistance than PTEN loss alone $(P=0.023$; Figure $2 \mathrm{C})$.

\section{Correlation of PTEN Status with Patients' Clinical Response to Trastuzumab-Based Therapy in Different Therapeutic Subgroups}

In the whole cohort, PTEN loss alone was not significantly associated with resistance to trastuzumab-based therapy $(P=0.081)$. The patients had varying treatment backgrounds, and we postulated that the predictive effect of PTEN status for trastuzumab response might be confounded by these different backgrounds. To examine this, we performed extended analyses of PTEN status and patients' clinical response to trastuzumab-based therapy after adjusting for the patients' treatment backgrounds.

First, we found that the rate of PTEN loss in tumors did not differ among different therapeutic subgroups of patients (see Supplemental Table S3 at http://ajp.amjpathol. org). Next, we performed a correlation analysis of PTEN status and trastuzumab response in different therapeutic subgroups. Among patients using trastuzumab as firstline therapy, PTEN loss alone was significantly associated with resistance to trastuzumab $(P=0.028)$ and more significantly correlated with shorter OS $(P=0.008)$ than in the whole cohort $(P=0.023)$. However, if the analysis was confined to the subgroup using trastuzumab as "other-line therapy" ( $\geq$ second-line therapy), there were no significant differences in trastuzumab response or OS between patients with PTEN-loss tumors and those with PTEN-normal tumors $(P=1.000$, $P=0.863$; Table 4). We also compared the whole cohort with the subgroup who received no prior che-

Table 4. Associations of PTEN Status with Clinical Outcomes after Trastuzumab-Based Therapy in Different Therapeutic Subgroups

\begin{tabular}{|c|c|c|c|c|}
\hline \multirow[b]{2}{*}{$\begin{array}{l}\text { Therapeutic } \\
\text { Subgroups }\end{array}$} & \multicolumn{3}{|c|}{ Response } & \multirow{2}{*}{$\begin{array}{c}\text { Overall } \\
\text { Survival } \\
P \text { Value } \\
\text { (Log Rank) }\end{array}$} \\
\hline & \multicolumn{2}{|c|}{$\mathrm{CR}+\mathrm{PR}$} & $\begin{array}{c}P \text { Value } \\
\text { (Fisher } \\
\text { Exact) }\end{array}$ & \\
\hline \multicolumn{5}{|c|}{ Ttzm as first-line therapy } \\
\hline PTEN loss & 24 & 22 & 0.028 & 0.008 \\
\hline PTEN normal & 31 & 10 & & \\
\hline \multicolumn{5}{|c|}{ Ttzm as other-line therapy } \\
\hline PTEN loss & 13 & 12 & 1.000 & 0.863 \\
\hline PTEN normal & 14 & 11 & & \\
\hline \multicolumn{5}{|c|}{ Without prior chemotherapy } \\
\hline PTEN loss & 21 & 22 & 0.030 & 0.013 \\
\hline PTEN normal & 35 & 13 & & \\
\hline \multicolumn{5}{|c|}{ With prior chemotherapy } \\
\hline PTEN loss & 12 & 11 & 1.000 & 0.922 \\
\hline PTEN normal & 9 & 7 & & \\
\hline
\end{tabular}

Ttzm indicates trastuzumab; $C R$, complete response; PR, partial response; SD, stable disease; $\mathrm{PD}$, progressive disease. 
Table 5. Correlation between PTEN Status and ER/PR Expression Status

\begin{tabular}{lcccc}
\hline Subgroup Variable & $\begin{array}{c}\text { PTEN } \\
\text { Loss }\end{array}$ & $\begin{array}{c}\text { PTEN } \\
\text { Normal }\end{array}$ & $\begin{array}{c}\text { Total } \\
\text { Value }\end{array}$ \\
\hline $\begin{array}{l}\text { ER-/PR- patients } \\
\text { Other patients }\end{array}$ & 42 & 27 & 69 & \\
$\quad$ (ER-/PR+ or & 27 & 36 & 63 & \\
$\quad$ ER+/PR- or & & & & \\
$\quad$ ER+/PR+) & & & & \\
Total & 69 & 63 & 132 & 0.039 \\
\hline
\end{tabular}

ER indicates estrogen receptor; PR, progesterone receptor.

motherapy before trastuzumab-based treatment and found that PTEN loss was significantly associated with trastuzumab resistance $(P=0.030)$ and shorter OS $(P=0.013)$ in patients who received no prior chemotherapy before trastuzumab-based treatment. However, PIK3CA mutations had no correlation with trastuzumab response in any subgroup (see Supplemental Table S4 at http://ajp.amjpathol.org).

\section{Associations of PTEN Status with Patients' Clinical Outcomes after Trastuzumab-Based Therapy in Different ER and PR Expression Subgroups}

Previous studies reported that the HER2 signaling pathway can cross talk with $\mathrm{ER},{ }^{4}$ and $\mathrm{ER}$ may play a role in resistance to HER2 inhibitors. ${ }^{34}$ To assess whether hormone receptor status involves in trastuzumab response, we performed trastuzumab response analyses based on hormone receptor status in patients' breast tumors. We found that neither ER status nor PR status was correlated with clinical outcomes in the whole cohort, but ER- patients had a trend toward PD after trastuzumab-based therapy $(P=0.099$, data not shown). Interestingly, PTEN loss was significantly associated with ER-/PR- status in this cohort $(P=0.039$; Table 5$)$. When we classified patients based on their ER-/PR - status, PTEN loss was found to be significantly associated with trastuzumab resistance $(P=0.029)$ and shorter OS $(P=0.033)$ in the ER-/PR- subgroup (Table 6).

\section{Discussion}

To our knowledge, this is the analysis of PI3K pathway activation (PTEN loss, PI3K mutation, PAKT+, and p-p70S6K +) in the largest cohort of HER2-overexpressing breast cancer patients who received trastuzumabbased therapy for metastatic disease. Our results demonstrated that the status of the four biomarkers (PTEN, PIK3CA, p-AKT, and p-p70S6K) did not significantly correlate with trastuzumab response when analyzed individually. Only PTEN loss was significantly correlated with shorter OS after trastuzumab-based therapy. PI3K pathway activation, defined as PTEN loss and/or PIK3CA mutation, significantly contributed to both worse response to trastuzumab and shorter OS. Although p-AKT and
Table 6. Association of PTEN Status with Clinical Outcomes after Trastuzumab-Based Therapy in Different ER/ PR Expression Subgroups

\begin{tabular}{|c|c|c|c|c|}
\hline \multirow[b]{2}{*}{ Subgroup Variable } & \multicolumn{3}{|c|}{ Response } & \multirow[b]{2}{*}{$\begin{array}{c}\text { Overall } \\
\text { Survival } \\
P \text { Value } \\
\text { (Log Rank) }\end{array}$} \\
\hline & $\begin{array}{c}\mathrm{CR}+\mathrm{PR} \\
+\mathrm{SD}\end{array}$ & PD & $\begin{array}{c}P \\
\text { Value } \\
\text { (Fisher } \\
\text { Exact) }\end{array}$ & \\
\hline \multicolumn{5}{|l|}{ ER-/PR- patients } \\
\hline PTEN loss & 18 & 24 & 0.029 & 0.033 \\
\hline PTEN normal & 19 & 8 & & \\
\hline \multicolumn{5}{|l|}{$\begin{array}{l}\text { Other patients } \\
\quad(\mathrm{ER}-/ \mathrm{PR}+\text { or } \\
\text { ER+/PR }- \text { or } \\
\text { ER+/PR+) }\end{array}$} \\
\hline PTEN Ioss & 17 & 10 & 0.795 & 0.348 \\
\hline PTEN normal & 24 & 12 & & \\
\hline
\end{tabular}

ER indicates estrogen receptor; $\mathrm{PR}$, progesterone receptor; $\mathrm{CR}$, complete response; PR, partial response; SD, stable disease; PD, progressive disease.

p-p70S6K status had limited correlation with trastuzumab response and OS in this cohort, when combined with PTEN status, PTEN loss/p-AKT+ and PTEN loss $/ \mathrm{p}$ p70S6K + were significantly related to shorter OS, with more power than PTEN loss alone. Remarkably, PTEN loss was significantly associated with trastuzumab resistance in patients who received trastuzumab as their firstline therapy or those who did not receive prior chemotherapy and in patients whose tumors were ER-/PR-. These data suggest that PI3K pathway activation through PTEN loss or PIK3CA mutation plays a pivotal role in resistance to trastuzumab-based therapy in patients with HER2-overexpressing MBC, particularly in patients who received trastuzumab as first-line therapy, those who had not received prior chemotherapy, and those with ER-l PR-tumors).

Trastuzumab resistance is becoming increasingly important in the clinic, as understanding mechanisms of resistance may lead to novel therapeutic approaches to overcome resistance. Although trastuzumab may decrease the risk of recurrence by $50 \%$ in patients with HER2-positive early-stage breast cancer, approximately $15 \%$ of patients develop metastatic disease despite adjuvant trastuzumab therapy. ${ }^{35}$ Identifying the most relevant and effective predictive biomarkers of trastuzumab response is essential for selecting subgroups of patients who can benefit from trastuzumab-based therapy and patients who may need additional or alternative therapies. Multiple genetic and molecular alterations have been found to contribute to trastuzumab resistance, among which PI3K pathway constitutive activation is one of the most important. ${ }^{11,25,36}$

In this study, PTEN-loss and PIK3CA mutations were found in $51.8 \%$ and $21.8 \%$, respectively, of the patients with HER2-positive MBC, which is consistent with previous reports. ${ }^{11,37,38}$ Taking into consideration PTEN-loss and PIK3CA mutation together, we found that patients having tumors without PI3K pathway activation received more benefit from trastuzumab-based therapy than patients with PI3K pathway activation tumors; patients having tumors without PI3K pathway activation had longer 
OS and better responses to trastuzumab. This observation indicates that evaluating both PTEN loss and PIK3CA mutations, or PTEN loss and PI3K downstream signaling events ( $p$-AKT or p-p70S6K), will better determine which patients are more likely to benefit from trastuzumabbased therapy in the metastatic setting. Our results also indicate that the PI3K pathway plays a pivotal role in conferring trastuzumab resistance, which is consistent with the results of previous studies. ${ }^{11,25}$ On the basis of the critical contribution of the PI3K pathway in human cancer, it is rational that combining trastuzumab with PI3K/AKT inhibitors could overcome trastuzumab resistance. ${ }^{39}$

Previous studies showed that PTEN loss led to increased PI3K/AKT signaling and that HER2-overexpressing breast cancer cell lines with low PTEN expression were more resistant to trastuzumab. ${ }^{11,25,26,36}$ Additionally, an unbiased large-scale RNA interference screen on genes involved in trastuzumab resistance in breast cancer identified PTEN as the only modulator of trastuzumab sensitivity. ${ }^{25}$ In our study, although PTEN loss alone was borderline significant with trastuzumab response $(P=$ $0.081)$, there was a trend toward lower response rates and significantly shorter OS $(P=0.023)$ after trastuzumab-based therapy in patients with tumors of low PTEN expression. However, PIK3CA mutation status did not correlate with either response or survival after trastuzumab treatment, suggesting that the role of PTEN loss may be more important than PIK3CA mutations in conferring trastuzumab resistance.

Our data indicated that the relationship between PTEN status and trastuzumab response and OS might be confounded by the patients' treatment backgrounds, as these were highly varied. Therefore, it was necessary to consider the patients' treatment background when analyzing the role of PTEN status in trastuzumab resistance. Remarkably, in patients who were treated with trastuzumab as first-line therapy or who did not receive prior chemotherapies, PTEN loss alone was highly associated with a worse response to trastuzumab-based therapy $(P=0.028 ; P=0.030)$. Even more significantly, PTEN loss strongly correlated with shorter OS $(P=0.008 ; P=$ $0.013)$ in these subgroups of patients. On the other hand, PIK3CA mutations had no significant correlation with either response or OS in any subgroup. These results suggest that i) prior treatments might alter PTEN expression levels or functions; ii) prior therapies might affect the activation level of PI3K pathway, indirectly altering the PTEN status; or iii) prior therapies might modify alternative signaling pathways related to PTEN expression. These data support the notion that PTEN loss plays a central role in trastuzumab resistance, while PIK3CA mutations may not be as critical, which is different from what has been previously reported. ${ }^{25}$ In addition, the mixed treatment backgrounds of the patients might be the reason for the different results in our study compared to other clinical studies on PTEN conferring trastuzumab resistance. In our study, only 55 patients' HER2-positive MBCs were analyzed for PIK3CA mutations. Therefore, interpretation of our results of PIK3CA mutations and trastuzumab resistance might be limited by the relatively small population size, and a larger cohort is needed to verify our results in the future. Our data indicated that patients with PTEN-normal tumors received the greatest benefit from first-line trastuzumab-based therapy.

PTEN loss or PIK3CA mutations direct PI3K-dependent tumorigenesis largely through activation of AKT kinase. Patients with PTEN-loss/p-AKT+ tumors or PTEN-loss/pp70S6K + tumors had significantly shorter OS than those with PTEN-normal/p-AKT- tumors or PTEN-normal/pp70S6K-tumors, suggesting the importance of an activated $\mathrm{PI} 3 \mathrm{~K}$ pathway in trastuzumab resistance. However, the two downstream biomarkers ( $p$-AKT, p-p70S6K) alone had limited value as indicators of trastuzumab resistance as noted in previous studies. ${ }^{27,28}$ Recently, it was reported that PI3K may promote cancer progression through an AKT-independent pathway, such as phosphoinositide-dependent kinase 1 pathway. ${ }^{40}$ In our cohort, there was no correlation between PTEN Ioss/PIK3CA mutations and p-AKT/p-p70S6K (data not shown), suggesting that PIK3CA mutations might not be sufficient to induce AKT and p70S6K phosphorylations. It is also possible that other upstream signaling events are contributing to $\mathrm{p}-\mathrm{AKT} / \mathrm{p}-\mathrm{p} 70 \mathrm{~S} 6 \mathrm{~K}$. Additionally, only five cases of PIK3CA mutations were seen among 29 cases of breast cancer patients with low PTEN levels, suggesting that PIK3CA mutation and PTEN loss may have distinct roles in trastuzumab response. Further studies are needed to address whether the phosphoinositide-dependent kinase 1 pathway or other pathways take part in trastuzumab resistance in addition to PTEN loss and PIK3CA mutations.

Concomitant up-regulation of PI3K/AKT signaling in HER2-overexpressing breast cancers can suppress ER and PR transcriptional activity. ${ }^{22,41,42}$ For patients who have HER2-positive and hormone receptor-negative breast tumors, finding a biomarker to predict trastuzumab resistance is even more important. In the present study, we found a correlation between hormone receptor negative status and PTEN deficiency $(P=0.039)$. Moreover, in the ER-/PR - group, PTEN expression alone was significantly correlated with both poor response $(P=$ 0.029 ) and shorter OS after trastuzumab-based therapy $(P=0.033)$, whereas in the $\mathrm{ER}+/ \mathrm{PR}+$ group, it was not. Our limited data are consistent with findings from other studies that loss of heterozygosity at the PTEN locus contributed to PR reduction and that PTEN functions might be different in ER+ versus ER- tumors. ${ }^{22,29,41,42}$ The data also suggest that abnormal expression of ER or PR might be involved in trastuzumab response. Further studies are needed to investigate whether ER and PR signaling deregulation is involved in mediating PTEN loss-induced trastuzumab resistance.

In conclusion, we have demonstrated that PI3K pathway activation (defined as PTEN Ioss, PIK3CA mutation, $\mathrm{p}-\mathrm{AKT}+$, and $\mathrm{p}-\mathrm{p} 70 \mathrm{~S} 6 \mathrm{~K}+$ ) contributes to trastuzumab resistance and short OS in patients with HER2-positive MBC treated by trastuzumab-based therapies. Remarkably, PTEN loss itself may serve as a predictor of resistance to trastuzumab in patients who receive trastuzumab as first-line therapy and in patients with negative hormone receptor status. Further studies are necessary 
to dissect the detailed mechanism of PTEN loss-mediated trastuzumab resistance and to help select the best treatment for individual patients.

\section{Acknowledgments}

We thank Sumaiyah Rehman, Dr. Shau-Hsuan Li, and Dr. Jing Lu for their valuable comments on this manuscript. We also thank Michael Worley and Alyson Todd (Department of Scientific Publication, MDACC) for their professional editing of the manuscript.

\section{References}

1. Slamon DJ, Clark GM, Wong SG, Levin WJ, Ullrich A, McGuire WL: Human breast cancer: correlation of relapse and survival with amplification of the HER-2/neu oncogene. Science 1987, 235:177-182

2. Rohan TE, Hartwick W, Miller AB, Kandel RA: Immunohistochemical detection of c-erbB-2 and p53 in benign breast disease and breast cancer risk. J Natl Cancer Inst 1998, 90:1262-1269

3. Hynes NE, MacDonald G: ErbB receptors and signaling pathways in cancer. Curr Opin Cell Biol 2009, 21:177-184

4. Kurokawa $\mathrm{H}$, Arteaga CL: ErbB (HER) receptors can abrogate antiestrogen action in human breast cancer by multiple signaling mechanisms. Clin Cancer Res 2003, 9:511S-515S

5. Emens LA: Trastuzumab: targeted therapy for the management of HER-2/neu-overexpressing metastatic breast cancer. Am J Ther 2005, 12:243-253

6. Romond EH, Perez EA, Bryant J, Suman VJ, Geyer CE Jr, Davidson NE, Tan-Chiu E, Martino S, Paik S, Kaufman PA, Swain SM, Pisansky TM, Fehrenbacher L, Kutteh LA, Vogel VG, Visscher DW, Yothers G, Jenkins RB, Brown AM, Dakhil SR, Mamounas EP, Lingle WL, Klein PM, Ingle JN, Wolmark N: Trastuzumab plus adjuvant chemotherapy for operable HER2-positive breast cancer. N Engl J Med 2005, 353:1673-1684

7. Piccart-Gebhart MJ, Procter M, Leyland-Jones B, Goldhirsch A, Untch M, Smith I, Gianni L, Baselga J, Bell R, Jackisch C, Cameron D, Dowsett M, Barrios CH, Steger G, Huang CS, Andersson M, Inbar M, Lichinitser M, Lang I, Nitz U, Iwata H, Thomssen C, Lohrisch C, Suter TM, Ruschoff J, Suto T, Greatorex V, Ward C, Straehle C, McFadden E, Dolci MS, Gelber RD: Trastuzumab after adjuvant chemotherapy in HER2-positive breast cancer. N Engl J Med 2005, 353:1659-1672

8. Esteva FJ, Valero V, Booser D, Guerra LT, Murray JL, Pusztai L, Cristofanilli M, Arun B, Esmaeli B, Fritsche HA, Sneige N, Smith TL, Hortobagyi GN: Phase II study of weekly docetaxel and trastuzumab for patients with HER-2-overexpressing metastatic breast cancer. J Clin Oncol 2002, 20:1800-1808

9. Clynes RA, Towers TL, Presta LG, Ravetch JV: Inhibitory Fc receptors modulate in vivo cytoxicity against tumor targets. Nat Med 2000, 6:443-446

10. Molina MA, Codony-Servat J, Albanell J, Rojo F, Arribas J, Baselga J: Trastuzumab (herceptin), a humanized anti-Her2 receptor monoclonal antibody, inhibits basal and activated Her2 ectodomain cleavage in breast cancer cells. Cancer Res 2001, 61:4744-4749

11. Nagata Y, Lan KH, Zhou X, Tan M, Esteva FJ, Sahin AA, Klos KS, Li P, Monia BP, Nguyen NT, Hortobagyi GN, Hung MC, Yu D: PTEN activation contributes to tumor inhibition by trastuzumab, and loss of PTEN predicts trastuzumab resistance in patients. Cancer Cell 2004, 6:117-127

12. Yakes FM, Chinratanalab W, Ritter CA, King W, Seelig S, Arteaga CL: Herceptin-induced inhibition of phosphatidylinositol-3 kinase and Akt Is required for antibody-mediated effects on p27, cyclin D1, and antitumor action. Cancer Res 2002, 62:4132-4141

13. Metro G, Mottolese M, Fabi A: HER-2-positive metastatic breast cancer: trastuzumab and beyond. Expert Opin Pharmacother 2008, 9:2583-2601

14. Nahta R, Yu D, Hung MC, Hortobagyi GN, Esteva FJ: Mechanisms of disease: understanding resistance to HER2-targeted therapy in human breast cancer. Nat Clin Pract Oncol 2006, 3:269-280
15. Esteva FJ, Yu D, Hung MC, Hortobagyi GN: Molecular predictors of response to trastuzumab and lapatinib in breast cancer. Nat Rev Clin Oncol 2010, 7:98-107

16. Mills GB, Kohn E, Lu Y, Eder A, Fang X, Wang H, Bast RC, Gray J, Jaffe R, Hortobagyi G: Linking molecular diagnostics to molecular therapeutics: targeting the PI3K pathway in breast cancer. Semin Oncol 2003, 30:93-104

17. Vivanco I, Sawyers CL: The phosphatidylinositol 3-Kinase AKT pathway in human cancer. Nat Rev Cancer 2002, 2:489-501

18. Hynes NE, Dey JH: PI3K inhibition overcomes trastuzumab resistance: blockade of ErbB2/ErbB3 is not always enough. Cancer Cell 2009, 15:353-355

19. Chan CT, Metz MZ, Kane SE: Differential sensitivities of trastuzumab (Herceptin)-resistant human breast cancer cells to phosphoinositide-3 kinase (PI-3K) and epidermal growth factor receptor (EGFR) kinase inhibitors. Breast Cancer Res Treat 2005, 91:187-201

20. Vazquez F, Sellers WR: The PTEN tumor suppressor protein: an antagonist of phosphoinositide 3-kinase signaling. Biochim Biophys Acta 2000, 1470:M21-M35

21. Bachman KE, Argani P, Samuels Y, Silliman N, Ptak J, Szabo S, Konishi H, Karakas B, Blair BG, Lin C, Peters BA, Velculescu VE, Park $\mathrm{BH}$ : The PIK3CA gene is mutated with high frequency in human breast cancers. Cancer Biol Ther 2004, 3:772-775

22. Saal LH, Holm K, Maurer M, Memeo L, Su T, Wang X, Yu JS, Malmstrom PO, Mansukhani M, Enoksson J, Hibshoosh H, Borg A, Parsons R: PIK3CA mutations correlate with hormone receptors, node metastasis, and ERBB2, and are mutually exclusive with PTEN loss in human breast carcinoma. Cancer Res 2005, 65:2554-2559

23. Perez-Tenorio G, Alkhori L, Olsson B, Waltersson MA, Nordenskjold B, Rutqvist LE, Skoog L, Stal O: PIK3CA mutations and PTEN loss correlate with similar prognostic factors and are not mutually exclusive in breast cancer. Clin Cancer Res 2007, 13:3577-3584

24. Isakoff SJ, Engelman JA, Irie HY, Luo J, Brachmann SM, Pearline RV, Cantley LC, Brugge JS: Breast cancer-associated PIK3CA mutations are oncogenic in mammary epithelial cells. Cancer Res 2005, 65:10992-11000

25. Berns K, Horlings HM, Hennessy BT, Madiredjo M, Hijmans EM, Beelen K, Linn SC, Gonzalez-Angulo AM, Stemke-Hale K, Hauptmann M, Beijersbergen RL, Mills GB, van de Vijver MJ, Bernards R: A functional genetic approach identifies the PI3K pathway as a major determinant of trastuzumab resistance in breast cancer. Cancer Cell 2007, 12:395-402

26. Junttila TT, Akita RW, Parsons K, Fields C, Lewis Phillips GD, Friedman LS, Sampath D, Sliwkowski MX: Ligand-independent HER2/ HER3/PI3K complex is disrupted by trastuzumab and is effectively inhibited by the PI3K inhibitor GDC-0941. Cancer Cell 2009, 15:429-440

27. Yonemori K, Tsuta K, Shimizu C, Hatanaka Y, Hashizume K, Ono M, Kouno T, Ando M, Tamura K, Katsumata N, Hasegawa T, Kinoshita T, Fujiwara Y: Immunohistochemical expression of PTEN and phosphorylated Akt are not correlated with clinical outcome in breast cancer patients treated with trastuzumab-containing neo-adjuvant chemotherapy. Med Oncol 2008, 26:344-349

28. Gori S, Sidoni A, Colozza M, Ferri I, Mameli MG, Fenocchio D, Stocchi L, Foglietta J, Ludovini V, Minenza E, De Angelis V, Crino L: EGFR, pMAPK, pAkt and PTEN status by immunohistochemistry: correlation with clinical outcome in HER2-positive metastatic breast cancer patients treated with trastuzumab. Ann Oncol 2009, 20:648-654

29. Klos KS, Wyszomierski SL, Sun M, Tan M, Zhou X, Li P, Yang W, Yin G, Hittelman WN, Yu D: ErbB2 increases vascular endothelial growth factor protein synthesis via activation of mammalian target of rapamycin/p70S6K leading to increased angiogenesis and spontaneous metastasis of human breast cancer cells. Cancer Res 2006, 66:2028-2037

30. Zhou Y, Pan Y, Zhang S, Shi X, Ning T, Ke Y: Increased phosphorylation of p70 S6 kinase is associated with HPV16 infection in cervical cancer and esophageal cancer. Br J Cancer 2007, 97:218-222

31. Shousha S: Oestrogen receptor status of breast carcinoma: allred/ $/ H$ score conversion table. Histopathology 2008, 53:346-347

32. Frank TS, Deffenbaugh AM, Reid JE, Hulick M, Ward BE, Lingenfelter B, Gumpper KL, Scholl T, Tavtigian SV, Pruss DR, Critchfield GC: Clinical characteristics of individuals with germline mutations in BRCA1 and BRCA2: analysis of 10,000 individuals. J Clin Oncol 2002, 20:1480-1490 
33. Jaffe CC: Measures of response: rECIST. WHO, and new alternatives $\mathrm{J}$ Clin Oncol 2006, 24:3245-3251

34. Singer CF, Kostler WJ, Hudelist G: Predicting the efficacy of trastuzumab-based therapy in breast cancer: current standards and future strategies. Biochim Biophys Acta 2008, 1786:105-113

35. Mariani G, Fasolo A, De Benedictis E, Gianni L: Trastuzumab as adjuvant systemic therapy for HER2-positive breast cancer. Nat Clin Pract Oncol 2009, 6:93-104

36. Fujita T, Doihara H, Kawasaki K, Takabatake D, Takahashi H, Washio K, Tsukuda K, Ogasawara Y, Shimizu N: PTEN activity could be a predictive marker of trastuzumab efficacy in the treatment of ErbB2overexpressing breast cancer. Br J Cancer 2006, 94:247-252

37. Depowski PL, Rosenthal SI, Ross JS: Loss of expression of the PTEN gene protein product is associated with poor outcome in breast cancer. Mod Pathol 2001, 14:672-676

38. Xia W, Husain I, Liu L, Bacus S, Saini S, Spohn J, Pry K, Westlund R, Stein SH, Spector NL: Lapatinib antitumor activity is not dependent upon phosphatase and tensin homologue deleted on chromosome 10 in ErbB2-overexpressing breast cancers. Cancer Res 2007. 67:1170-1175

39. Lu CH, Wyszomierski SL, Tseng LM, Sun MH, Lan KH, Neal CL, Mills
GB, Hortobagyi GN, Esteva FJ, Yu D: Preclinical testing of clinically applicable strategies for overcoming trastuzumab resistance caused by PTEN deficiency. Clin Cancer Res 2007, 13:5883-5888

40. Vasudevan KM, Barbie DA, Davies MA, Rabinovsky R, McNear CJ, Kim JJ, Hennessy BT, Tseng H, Pochanard P, Kim SY, Dunn IF, Schinzel AC, Sandy P, Hoersch S, Sheng Q, Gupta PB, Boehm JS, Reiling JH, Silver S, Lu Y, Stemke-Hale K, Dutta B, Joy C, Sahin AA, Gonzalez-Angulo AM, Lluch A, Rameh LE, Jacks T, Root DE, Lander ES, Mills GB, Hahn WC, Sellers WR, Garraway LA: AKT-independent signaling downstream of oncogenic PIK3CA mutations in human cancer. Cancer Cell 2009, 16:21-32

41. Perren A, Weng LP, Boag AH, Ziebold U, Thakore K, Dahia PL, Komminoth P, Lees JA, Mulligan LM, Mutter GL, Eng C: Immunohistochemical evidence of loss of PTEN expression in primary ductal adenocarcinomas of the breast. Am J Pathol 1999, 155:1253-1260

42. Tokunaga E, Oki E, Kimura Y, Yamanaka T, Egashira A, Nishida K, Koga T, Morita M, Kakeji Y, Maehara Y: Coexistence of the loss of heterozygosity at the PTEN locus and HER2 overexpression enhances the Akt activity thus leading to a negative progesterone receptor expression in breast carcinoma. Breast Cancer Res Treat 2007, 101:249-257 